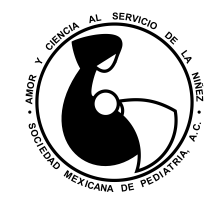

Revista Mexicana de
ARTí́CULO ORIGINAL

Vol. 86, No. 4 • Julio-Agosto 2019

pp 143-146

Recibido: 18/10/2018

Aceptado: 06/04/2019

dx.doi.org/10.35366/SP194C

\title{
Neurodesarrollo en niños con cardiopatía congénita a los 30 meses de edad
}

\author{
Viviana Mendoza-Sánchez, ${ }^{1, *}$ Laura Eugenia Hernández-Negrete, ${ }^{2}$ \\ Manuel Cázarez-Ortiz, ${ }^{3}$ Edith González, ${ }^{4}$ Martina Angélica Guido-Campuzano ${ }^{5}$ \\ ${ }^{1}$ Residente de $5^{\circ}$ año de Neonatología; ${ }^{2}$ Médico Neonatólogo; ${ }^{3}$ Profesor titular del curso de Neonatología; \\ ${ }^{4}$ Jefe del Servicio de Neonatología, ${ }^{5}$ Médico adscrito a Neonatología. Centro Médico Nacional 20 de Noviembre, ISSSTE. \\ Ciudad de México, México.
}

\begin{abstract}
RESUMEN
Objetivo: Describir el neurodesarrollo de pacientes con cardiopatía congénita compleja (CCC) a quienes se les realizó cirugía cardiaca en el periodo neonatal. Material y métodos: Mediante un estudio longitudinal, observacional y retrospectivo incluimos 14 pacientes, evaluando el neurodesarrollo por medio de la escala de desarrollo infantil Bayley II a la edad de 30 meses. Resultados: La edad promedio al momento de la cirugía de 19 días. La cardiopatía más frecuente fue coartación aórtica (43\%). Del total, $29 \%$ tuvo desarrollo cognitivo bajo, desarrollo psicomotriz en el $50 \%$ anormal. Conclusiones: Los pacientes pediátricos con CCC después de la cirugía tienen alto riesgo de presentar déficit neurológico, con predominio en el área psicomotriz.
\end{abstract}

Palabras clave: Neurodesarrollo, cardiopatía congénita, déficit neurológico.

\section{INTRODUCCIÓN}

La incidencia de las cardiopatías congénitas oscila entre ocho y 12 por cada 1,000 recién nacidos vivos. ${ }^{1}$ Se estima que entre 30,000 y 40,000 niños nacen cada año con cardiopatía congénita compleja

\footnotetext{
* Correspondencia: VMS, vyp26-@hotmail.es

Conflicto de intereses: Los autores declaran que no tienen. Citar como: Mendoza-Sánchez V, Hernández-Negrete LE, Cázarez-Ortiz M, González E, Guido-Campuzano MA. Neurodesarrollo en niños con cardiopatía congénita a los 30 meses de edad. Rev Mex Pediatr. 2019; 86(4):143-146.

[Neurodevelopment in children with congenital heart disease at 30 months of age]
}

\begin{abstract}
Objective: To describe the neurodevelopmental status of patients with complex congenital heart disease (CCHD), who underwent cardiac surgery in the neonatal period. Material and methods: Through a longitudinal, observational and retrospective study, we included 14 patients. Neurodevelopment was assessed through the Bayley II child development scale, at the age of 30 months. Results: The average age at the time of surgery was 19 days. The most frequent heart disease was aortic coarctation (43\%). Of the total, $29 \%$ had low cognitive development, and $50 \%$ with abnormal psychomotor development. Conclusions: Pediatric patients with CCHD after surgery have a high risk of presenting neurological deficit, predominantly in the psychomotor area.
\end{abstract}

Keywords: Neurodevelopment, congenital heart disease, neurological deficit.

(CCC) en Estados Unidos de Norteamérica (EUA), de los cuales, aproximadamente un tercio requiere intervención quirúrgica durante el primer año de vida. En estos pacientes las discapacidades son comunes y afectan aproximadamente a la mitad de los supervivientes. ${ }^{2}$

Diversos estudios han demostrado una asociación entre CCC y el retraso del neurodesarrollo atribuido al riesgo de lesión cerebral durante la cirugía cardiaca. Se ha descrito que desde antes de la cirugía ya existen problemas del desarrollo neurológico, tales como crisis convulsivas, hipotonía, hipertonía, asimetría motora, ausencia de succión, dificultad a la alimentación, anomalías de nervios craneales, letargo, inquietud, agitación y autismo. ${ }^{3}$ 
Las lesiones cerebrales durante el desarrollo pueden resultar en déficits cognitivos, conductuales y psicológicos. Los avances en la cirugía cardiaca han mejorado las tasas de supervivencia; sin embargo, estos niños siguen siendo susceptibles a un espectro de deficiencias neurológicas. ${ }^{4}$ Por ejemplo, los niños con CCC tienen puntajes más bajos en las pruebas de funcionamiento adaptativo, déficit de lenguaje expresivo y déficit motor (20-50\%), así como dificultades de comunicación social. $^{5}$

Los efectos de la CCC y la cirugía en el neurodesarrollo dependen de diversos factores, incluyendo la complejidad de la lesión, las comorbilidades antes de la cirugía, además de lo que ocurre en el trans y postoperatorio. ${ }^{6}$ Los pacientes en quienes se utiliza circulación extracorpórea (CEC) pueden presentar convulsiones, hemorragia o infarto cerebral, y muerte cerebral. En general, se conoce que existe menor probabilidad de daño neurológico cuando el tiempo de paro cardiaco es inferior a 60 minutos y el tiempo de CEC es menor a 90 minutos. $^{7}$

La escala de desarrollo infantil Bayley II (BSDII) es el estándar de oro internacional para evaluar el neurodesarrollo de niños y niñas entre dos meses y 30 meses de vida. Los ítems de la escala evalúan la maduración de las habilidades cognitivas y motoras, tomando como referente el comportamiento de sujetos normales. ${ }^{8,9}$ Con la identificación temprana de las alteraciones del neurodesarrollo y las discapacidades, los niños tienen una mejor oportunidad para lograr su máximo potencial. ${ }^{10}$

El objetivo de este estudio es describir el neurodesarrollo de pacientes pediátricos con CCC después de la cirugía.

\section{MATERIAL Y MÉTODOS}

Se analizaron 14 pacientes con diagnóstico de CCC y con el antecedente de cirugía cardiaca correctiva o paliativa, realizada durante el periodo neonatal. Estos pacientes acudieron a la consulta de seguimiento longitudinal del Centro Médico Nacional 20 de Noviembre, con revisiones mensuales durante el primer año de vida, y posteriormente de manera semestral hasta los dos años y seis meses de edad. Durante estas revisiones se llevó a cabo la evaluación del desarrollo con la escala Bayley II por el médico neonatólogo.

Es de señalar que en estos pacientes se documentó la ausencia de daño neurológico previo a la cirugía cardiaca, y tampoco tenían antecedentes de asfixia perinatal, malformaciones neurológicas, neuroinfección, crisis convulsivas, hemorragia intracraneal, trauma craneoencefálico o cualquier evento que originara o contribuyera a problemas del neurodesarrollo, como síndromes genéticos.

Análisis estadístico. Los resultados se presentan como estadística descriptiva, con promedio y desviación estándar para las variables numéricas. Las cualitativas con frecuencias absolutas y porcentajes.

\section{RESULTADOS}

Fueron 10 (71\%) hombres y cuatro (29\%) mujeres. La edad al momento de la cirugía realizada fue en promedio 19 días. Los tipos de CCC se muestran en la Figura 1. La coartación crítica de aorta fue la más común, alcanzó la frecuencia de seis (43\%). La cirugía correctiva fue la más frecuente en ocho pacientes (57\%).

La descripción de los valores obtenidos del índice de desarrollo mental en cada paciente se muestra en la Figura 2. El promedio del índice en la muestra fue de $104.0 \pm 29.5$, con una variación de 50 a 159 . Tomando como valor de corte 84 (lo que corresponde a normal bajo), tres pacientes tuvieron nivel de neurodesarrollo bajo (21-4\%) y en 11 (78.6\%) con un nivel normal.

Con respecto al índice de desarrollo psicomotor, los datos de los 14 pacientes se presentan en la Figura 3. El promedio fue de $87.4 \pm 23.6$ puntos, con una variación de 50 a 140. Tomando el mismo nivel de corte (84), a siete (50\%) pacientes se consideró como bajo y el resto normal.

Por último, se detectaron 11 (78.6\%) pacientes con algún tipo de discapacidad; cinco con alteraciones en



Figura 1: Distribución de los 14 pacientes por tipo de cardiopatía. 


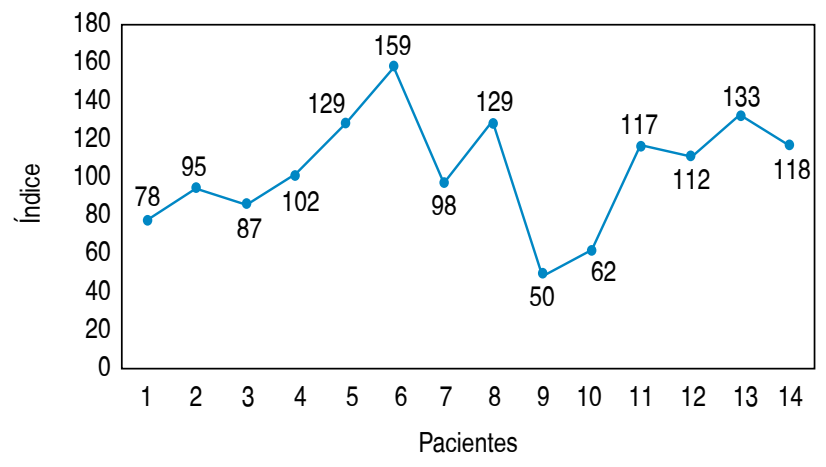

Figura 2: Índice de desarrollo mental de 14 pacientes en torno al valor de la normalidad $(\geq 84)$.

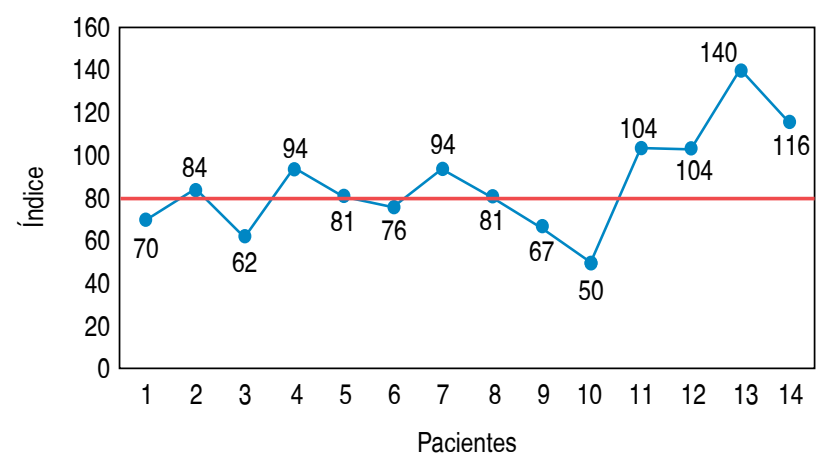

Figura 3: Índice del desarrollo psicomotriz de 14 pacientes en torno al valor de la normalidad $(\geq 84)$.

psicomotricidad (36\%), tres pacientes con afección en el leguaje y otros tres con retraso global de neurodesarrollo (21\%).

\section{DISCUSIÓN}

Más allá de la supervivencia, es motivo de gran interés conocer el pronóstico funcional de los pacientes con cardiopatía congénita. Marino y colaboradores en el estudio sobre el neurodesarrollo en niños con CCC, revisaron la literatura disponible en Medline con la información publicada entre 1966 y 2011. Encontraron que estos niños tienen mayor riesgo de presentar trastornos en el neurodesarrollo, por lo que recomiendan la vigilancia periódica y evaluación durante la infancia, a fin de otorgar intervenciones apropiadas para maximizar el potencial de desarrollo global de estos niños. ${ }^{10}$

Como el presente trabajo, la mayoría de los estudios previos han investigado los resultados después de la cirugía en niños con CCC, reportando cierto grado de deterioro persistente en la motricidad fina o gruesa. En la revisión sistemática de Snookes y su grupo ${ }^{11}$ se incluyeron 65 artículos, que estudiaron el desarrollo cognitivo o motor de los niños con CCC sometidos a cirugía antes de los seis meses de edad. En todos los estudios emplearon la escala de desarrollo infantil de Bayley; los resultados al año de edad revelaron un promedio del índice de desarrollo mental de 90.3 (intervalo de confianza al 95\% [IC95\%] 88.9-91.6) y para el índice de desarrollo psicomotor 78.1 (IC95\% 76.4-79.7). Estos estudios revelaron consistentemente retraso cognitivo y motor en los niños después de la cirugía; estos hallazgos son comparables a los obtenidos en el presente estudio.

Donofrio y colegas ${ }^{12}$, en el artículo de 2010 sobre el impacto de la CCC en el neurodesarrollo, informan que aunque el índice de coeficiente intelectual parece estar en rango normal para los sobrevivientes de CCC, las tasas de trastornos del neurodesarrollo siguen siendo significativas (microcefalia, hipotonía, irritabilidad, asimetría motora, succión ausente y crisis convulsivas).

Los avances en la medicina, incluyendo el diagnóstico y la evaluación prenatal, las innovaciones en las técnicas de cirugía cardiotorácica y las mejoras en el manejo perioperatorio han contribuido al aumento de la supervivencia de los recién nacidos con CCC, por lo que se deben hacer más esfuerzos para mejorar los resultados a largo plazo. 8,13

\section{REFERENCIAS}

1. Hoffman JI. The global burden of congenital heart disease. Cardiovasc J Afr. 2013; 24(4): 141-145.

2. Wernovsky G, Licht DJ. Neurodevelopmental outcomes in children with congenital heart disease-what can we impact? Pediatr Crit Care Med. 2016; 17(8 Suppl 1): S232.

3. Khalil A, Suff N, Thilaganathan B, Hurrell A, Cooper D, Carvalho JS. Brain abnormalities and neurodevelopmental delay in congenital heart disease: systematic review and meta-analysis. Ultrasound Obstet Gynecol. 2014; 43(1): 14-24.

4. Morton PD, Ishibashi N, Jonas RA. Neurodevelopmental abnormalities and congenital heart disease: insights into altered brain maturation. Circ Res. 2017; 120(6): 960-977.

5. Nattel SN, Adrianzen L, Kessler EC, Andelfinger G, Dehaes $M$, Côté-Corriveau $\mathrm{G}$ et al. Congenital heart disease and neurodevelopment: clinical manifestations, genetics, mechanisms, and implications. Can J Cardiol. 2017; 33(12): 1543-1555.

6. Massaro AN, El-dib M, Glass P, Aly H. Factors associated with adverse neurodevelopmental outcomes in infants with congenital heart disease. Brain Dev. 2008; 30(7): 437-446.

7. Lequier L, Joffe AR, Robertson CM, Dinu IA, Wongswadiwat $Y$, Anton NR et al. Two-year survival, mental, and motor outcomes after cardiac extracorporeal life support at less than five years of age. J Thorac Cardiovasc Surg. 2008; 136(4): 976-983.

8. Pérez-López J, de la Nuez AG, Martínez-Fuentes MT, DíazHerrero A, Sánchez-Caravaca J, Fernández-Rego FJ. Las 
escalas Bayley BSID-I frente a BSID-II como instrumento de evaluación en atención temprana. Anales de Psicología. 2012; 28(2): 484.

9. Bayley N. Escalas Bayley de desarrollo infantil. Madrid: TEA. 1977.

10. Marino BS, Lipkin PH, Newburger JW, Peacock G, Gerdes M,

Gaynor JW et al. Neurodevelopmental outcomes in children with congenital heart disease: evaluation and management: a scientific statement from the American Heart Association. Circulation. 2012; 126(9): 1143-1172.
11. Snookes SH, Gunn JK, Eldridge BJ, et al. A systematic review of motor and cognitive outcomes after early surgery for congenital heart disease. Pediatrics. 2010;125(4):e818-e827.

12. Donofrio MT, Massaro AN. Impact of congenital heart disease on brain developmental and developmental outcome. Int J Pediatric. 2010; 2010.

13. Gerdes M, Flynn T. Clinical assessment of neurobehavioral outcomes in infants and children with congenital heart disease. Progress Pediatr Cardiol. 2010; 29: 97-105. 